DOI: $10.15593 / 2224-9982 / 2015.43 .03$

УДК 621.45.037

И.А. Кривошеев, К.Е. Рожков, Н.Б. Симонов

Уфимский государственный авиационный технический

университет, Уфа, Россия

ИСПОЛЬЗОВАНИЕ МЕТОДОВ РАСЧЕТА

ХАРАКТЕРИСТИК РЕШЕТОК ПРОФИЛЕЙ

ПРИ МОДЕЛИРОВАНИИ И ПРОЕКТИРОВАНИИ

КОМПРЕССОРОВ ГАЗОТУРБИННЫХ ДВИГАТЕЛЕЙ

\begin{abstract}
Рассматриваются методы расчета и параметризованного представления характеристик решеток профилей, лопаточных венцов, многоступенчатых компрессоров в составе газотурбинных двигателей и газотурбинных установок. Показано, что такие модели требуются при проектировании компрессоров и анализе изменения их характеристик в эксплуатации. При этом управление ограниченным числом параметров позволяет проводить оптимизацию (подбор геометрии лопаточных венцов, проточной части, кинематических параметров - частоты вращения, скорости рабочего тела). Для получения параметризованных моделей может быть использовано предположение о качественном подобии закономерностей протекания аэродинамических характеристик решеток профилей, ступеней и многоступенчатых компрессоров (каскадов). Показано, что используемые в настоящее время известные эмпирические зависимости, полученные на основании обобщения большого числа экспериментов, могут быть уточнены с учетом новых представлений о рабочих процессах, происходящих в лопаточных машинах. Дополнительно могут быть использованы результаты численных экспериментов с применением 3DCAD/CAE-моделирования. Приведена оригинальная методика, предложенная авторами. В ней используется обнаруженная связь градиента наклона линейной части характеристики решеток профилей и соответствующей эквивалентной решетки тонких пластин, предложенная эмпирическая зависимость для последней, предложенный метод номограмм, зависимость, альтернативная универсальной кривой Л.Е. Ольштейна и В.Г. Процерова, поправки, учитывающие скорость натекания, «просадку» осевой скорости, диагональность, закрутку на входе. Показано, как строится граница помпажа и линия номинальных режимов (на характеристике не только венца и ступени, но и многоступенчатого компрессора). Предложенный метод позволяет получать универсальную обезразмеренную характеристику компрессоров, включая универсальную границу помпажа, экстраполировать напорные ветви, строить новые, уточнять границу помпажа при экспериментах. Разработанные методы использованы в созданной авторами системе имитационного повенцового моделирования компрессоров различных типов VENEC.
\end{abstract}

Ключевые слова: газотурбинный двигатель, компрессор, проточная часть, термодинамические и газодинамические параметры. 


\author{
I.A. Krivosheev, K.E. Rozhkov, N.B. Simonov
}

Ufa State Aviation Technical University, Ufa, Russian Federation

\title{
USING THE METHODS OF CALCULATING \\ THE CHARACTERISTICS OF PROFILE GRIDS IN SIMULATION AND DESIGN OF COMPRESSOR OF GAS-TURBINE ENGINES
}

\begin{abstract}
The methods of calculation and presentation of parameterized performance of profile grids, blade tips, multi-stage compressors as part of a turbine engine and gas turbine are considered. It is shown that such models are required in the design of compressors and analyzing their characteristics in operation. At the same time, managing a limited number of settings allows optimization (selection of the geometry of blade tips, airgas channel, the kinematic parameters - rate of rotation, the speed of the working fluid). For parameterized models can be used assumption of qualitative similarity of regularities of aerodynamic characteristics of airfoil cascades, stages and multistage compressors (stages). It is shown that the currently used known empirical relationships, derived from generalization of a large number of experiments can be refined with new ideas about working processes in turbomachinery. In addition, it can be used the results of numerical experiments with the use of 3D CAD/CAE-modeling. The original method proposed by the authors is presented. It uses the detected relationship of the gradient of slope of linear part of profile grid characteristics and corresponding equivalent of thin plates, proposed an empirical relationship for the latter, the proposed method of graph, alternative dependence for universal curve L.E. Olshteyn and V.G. Protserov, corrections accounting the inleakage rate, "drawdown" of axial velocity, swirling at the entrance. It is shown how to construct a boundary line of surge and nominal modes (not just on the characteristics of the blade tip and the stage, but also multistage compressor). The proposed method allows obtaining the dimensionless universal characteristics of compressors, including universal surge limit, extrapolation of pressure branches and building the new ones, elaboration of the border surge in experiments. Developed methods are used by the authors in the created system of simulation modeling for compressor blade tips of various types VENEC.
\end{abstract} parameters.

Keywords: gas-turbine engine, compressor, air-gas channel, thermodynamic and gas-dynamic

Анализ показывает, что в используемых программных комплексах (Concepts NREC [1], COMPRESSOR [2] и т.п.) при выборе параметров, оптимизации, подготовке начальных и граничных условий для трехмерного (3D) CAD/CAE-моделирования компрессоров применяются двумерные (2D) модели, в основе которых лежат эмпирические зависимости, полученные в работах [3-9] на основе обобщения многочисленных экспериментов и позволяющие уже на ранних этапах проектирования рассчитывать параметры и характеристики решеток профилей, лопаточных венцов, ступеней и многоступенчатых компрессоров. Однако сравнение этих зависимостей с экспериментальными данными показывает, что имеются существенные расхождения либо эти зависимости имеют ограниченную область адекватности.

Использование феноменологического подхода, асимптотического анализа, теоретических представлений и 3DCAD/CAE-моделирования 
позволило авторам уточнить известные зависимости, расширить область их применения, а также получить ряд новых важных для практики зависимостей. В широком диапазоне изменения угла натекания на входе в решетку $\left(0<\beta_{1}<180^{\circ}\right)$, что позволяет рассмотреть и «компрессорный», и «турбинный» режимы, режимы обычного течения и после срыва потока, а также обратного течения (при «хлопках» во время помпажа), характеристика любой решетки $\Delta \beta=f\left(\beta_{1}\right)$ вполне определенным образом располагается по отношению к идеальной линии $\Delta \beta=\beta_{2 л}-\beta_{1}$ (луч под углом $-45^{\circ}$ ) (рис. 1). Основными параметрами, от которых зависит протекание характеристики решетки профилей, являются «лопаточный» угол на выходе $\beta_{2 л}$, кривизна профиля $\theta$, относительное утолщение $\bar{c}_{m}$, густота решетки $b / t$ и скорость натекания $\lambda_{w 1}$.

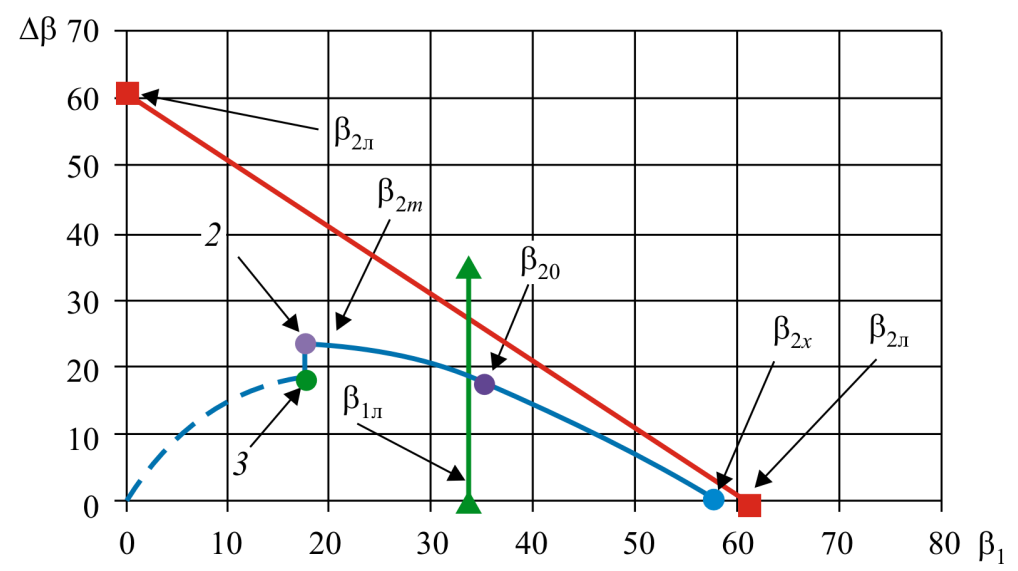

Рис. 1. Представление характеристики элементарной решетки в виде базовой зависимости угла отклонения от угла набегания

На характеристике решетки принято выделять точку «m», где достигается максимальный угол поворота $\Delta \beta_{m}=\Delta \beta_{\max }$. Известно, что левее этой точки «m» находится зона срыва потока (точки «2»и «3»). Точка на оси абсцисс обозначена « $x »$. Здесь $\Delta \beta_{x}=0$ и решетка имеет нулевой коэффициент теоретического напора: $\bar{H}_{T}=\bar{H}_{T x}=0$. В широком диапазоне эти точки (для «турбинного» режима) продублированы и помечены штрихом '.

Для параметризации характеристик решеток профилей использованы параметры в этих точках и показано, как они связаны с геометри- 
ей решетки и кинематическими параметрами. Так, например, на основе известного метода Л.Е. Ольштейна [6] характеристика любой решетки профилей в зоне автомодельности по скорости натекания $\left(\lambda_{w 1}<0,4\right)$ в виде $\bar{H}_{T}=f\left(\bar{c}_{a}\right)-$ зависимости коэффициента теоретического напора $\bar{H}_{T}$ решетки профилей от коэффициента расхода $\bar{c}_{a}$ должна вблизи номинального режима и правее его (при $\bar{c}_{a}>\bar{c}_{a 0}$ ) иметь линейный участок. Однако если следовать методу Л.Е. Ольштейна, то экстраполяция линейного участка введенных им функций $K_{1}$ и $K_{2}$ на ось ординат (определение значения $\bar{H}_{T}=\bar{H}_{T y}$ при $\bar{c}_{a}=0$ ) для любой ступени независимо от геометрии решеток и от относительного диаметра втулки дает значение $\bar{H}_{T y}=0,75$. Это противоречит экспериментальным данным и в общем случае вносит большую погрешность в расчеты.

Эксперименты по продувкам решеток профилей $[8,9]$ показывают, что параметр $\bar{H}_{T y}$ существенно зависит от геометрии решетки и скорости натекания потока $\lambda_{w 1}$. При этом авторами установлено, что в зоне автомодельности по $\lambda_{w 1}$ линейный участок базовой кривой $\bar{H}_{T}=f\left(\bar{c}_{a}\right)$ параллелен аналогичной линии для соответствующей решетки тонких пластин (с той же густотой $b / t$ и тем же «лопаточным» углом выхода $\beta_{2 л}$ ). Характерный параметр $\bar{H}_{T y}$ имеет два различных значения для участков характеристики: $\bar{H}_{T y 1}-$ до и $\bar{H}_{T y 2}-$ после возникновения срыва потока (рис. 2).

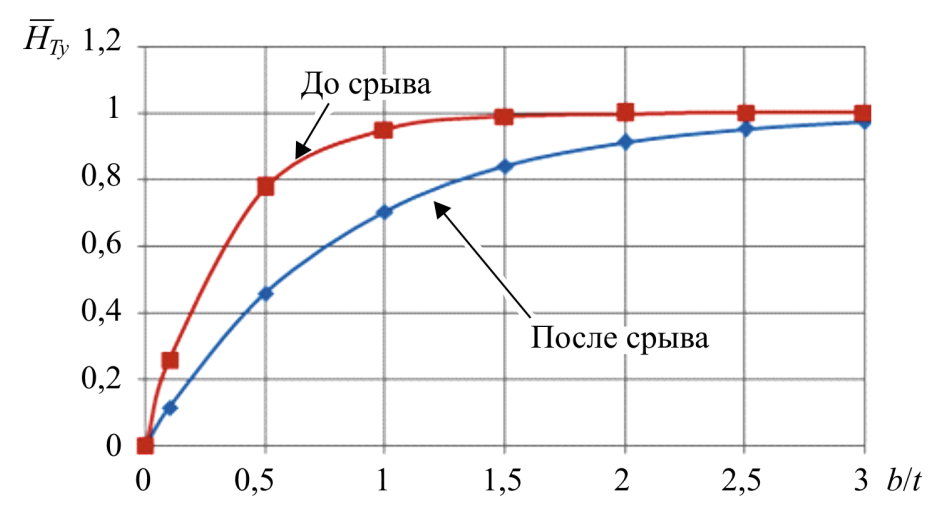

Рис. 2. Определение характерного параметра $\bar{H}_{T y}$ в зависимости от густоты решеток профилей 
Показано, что в обоих случаях для решетки тонких пластин справедлива экспоненциальная зависимость $\bar{H}_{T y(1,2)}$ от густоты решетки $b / t$ :

$$
\bar{H}_{T y(1,2)}=1-e^{-\frac{b / t}{T_{(1,2)}}} \text {. }
$$

При этом (путем численных и физических экспериментов) подобрано значение параметра $T: T_{1} \approx 0,42 \ldots 0,355$ - для характеристики на участке до начала срыва потока, $T_{2} \approx 0,82$ - для характеристики на участке после срыва потока. Это позволяет определять градиент наклона линейного (вблизи номинального режима «0») участка базовой (для автомодельности по скорости натекания $\left.\lambda_{w 1}<0,4\right)$ характеристики решетки тонких пластин как $\frac{\mathrm{d} \bar{H}_{T}}{\mathrm{~d} \bar{c}_{a}}=\frac{\bar{H}_{T y}}{\operatorname{tg} \beta_{2 л}}$. Здесь используется определенное для эквивалентной решетки тонких пластин значение $\bar{H}_{T y}$ и $\beta_{2 л}=\gamma$ (угол установки пластин в решетке).

Соответственно, для реальной решетки профилей таким образом определяется градиент наклона линейного участка характеристики до и после начала срыва потока $\frac{\mathrm{d} \bar{H}_{T}}{\mathrm{~d} \bar{c}_{a}}$. Дополнительно доказано, что при фронтальной продувке $\left(\beta_{1}=90^{\circ}\right)$ при заданном «лопаточном» угле на выходе $\beta_{2 л}$ и густоте решетки $b / t$ и при автомодельности по скорости натекания $\left(\lambda_{w 1}<0,4\right)$ угол на выходе $\beta_{2}=\beta_{2 N}$ в этом случае для всех таких решеток одинаков, и поэтому для них градиент наклона линейного участка характеристики также одинаков: $\frac{\mathrm{d} \bar{H}_{T}}{\mathrm{~d} \bar{c}_{a}}=\frac{1}{\operatorname{tg} \beta_{2 N}}$. Это позволило предложить простой метод получения характеристик с помощью фронтальных продувок при нулевой частоте вращения (неподвижных) не только для плоских решеток профилей, но и для рабочих колес. При этом возможно использование как современных лазерно-доплеровских методов визуализации, так и классических (дымарь, «метод шелковинок») при фиксации картин в видеорежиме и осреднении направления течения на выходе.

Установлено, что известная универсальная зависимость $\frac{\Delta \beta_{1}}{\Delta \beta_{10}}=f\left(\frac{\beta_{1}-\beta_{10}}{\Delta \beta_{0}}\right)$ [6], из которой следует, что вблизи номинального 
режима «0» градиент $\frac{\mathrm{d} \Delta \beta}{\mathrm{d} \beta_{1}}$ для всех решеток профилей примерно одинаков, имеет узкую область применения (рис. 3).

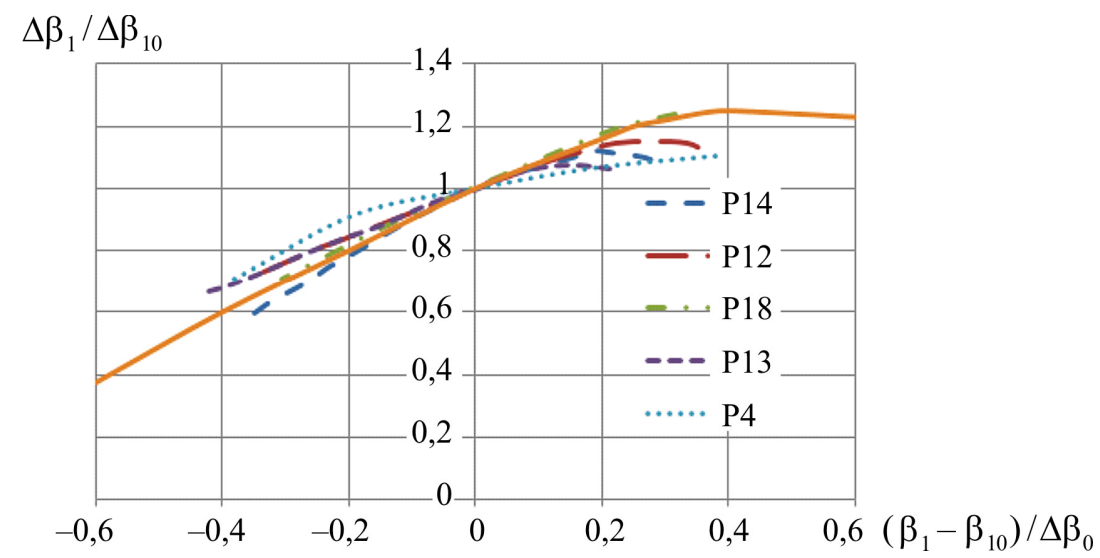

Рис. 3. Сравнение универсальной кривой номинальных режимов [6] с результатами продувок решеток конкретных профилей

Соответственно, то, что из этой зависимости следует (для точки «m», где $\left.\Delta \beta_{m}=\Delta \beta_{\max }\right) \frac{\beta_{1 m}-\beta_{10}}{\Delta \beta_{0}} \approx-0,4$, в общем случае не согласуется с результатами продувки не только решеток пластин [8, 9], но и обычных решеток профилей. Однако в ряде работ сделана попытка использовать это соотношение для построения границы помпажа на характеристиках ступеней и компрессоров, что может приводить к существенным погрешностям. В качестве более общего и более точного авторами предложено соотношение $\operatorname{tg} \beta_{1 m} / \operatorname{tg} \beta_{10} \approx 0,788$. Однако обнаружено, что и оно для втулочных решеток профилей (с большими кривизной $\theta$, углом установки $\gamma$ и утолщением $\bar{c}_{m}$ ) дает погрешность.

Из теоретических соображений в точке «m» $\frac{\mathrm{d} \Delta \beta}{\mathrm{d} \beta_{1}}=0$ и $\frac{\mathrm{d} \beta_{2}}{\mathrm{~d} \beta_{1}}=1$. Соответственно, в этой точке «m» базовая линия $\bar{H}_{T}=f\left(\bar{c}_{a}\right)$ любой решетки профилей должна касаться изолинии $\Delta \beta=\Delta \beta_{m}=$ const, которая строится при условии $\bar{H}_{T}=1-\operatorname{tg} \beta_{1} / \operatorname{tg}\left(\beta_{1}+\Delta \beta_{m}\right)$ в координатах $\left\{\bar{H}_{T}, \bar{c}_{a}\right\}$. На этом основании предложено строить семейство таких изолиний с пе- 
ременным шагом $\left(\Delta \beta_{i+1}=1,25 \cdot \Delta \beta_{i}\right)$, при этом каждая пара из таких изолиний соответственно характеризует номинальные режимы «0» и максимальные «m» для конкретной решетки. Это позволило для построения базовой линии $\bar{H}_{T}=f\left(\bar{c}_{a}\right)$ характеристики любой решетки профилей использовать метод номограмм.

Рассмотрен вопрос определения параметров на номинальном «0» режиме решетки профилей (в зависимости от геометрии решетки, направления и скорости натекания потока). Анализ показал, что наиболее точные результаты дает совместное использование зависимостей Бойса [2] и А.П. Комарова [2] (с поправкой на $\lambda_{w 1}$ ). Кроме того, для уточнения известной эмпирической зависимости Хауэлла [5] для определения номинальных режимов «0» авторами вместо «лучей Хауэлла» построено более точное семейство кривых.

Показано, что в отличие от метода Л.Н. Ольштейна (предложенного для ступеней) характеристики решеток профилей расслаиваются не по приведенной окружной скорости $\lambda_{u}$, а по приведенной скорости натекания (в относительном движении) $\lambda_{w 1}$. Для выделения соответствующих поправок выведена (при отсутствии закрутки на входе $\alpha_{1}=90^{\circ}$ ) взаимная зависимость параметров $\lambda_{w 1}, \lambda_{u}$ и $\bar{c}_{a}$.

Обнаружено, что известные формулы [2] для определения параметров решеток профилей на номинальном режиме непригодны для решеток профилей с малой или нулевой кривизной. Это ограничивает возможность расчета характеристик решеток пластин (в завихрителях камер сгорания газотурбинных двигателей) и решеток слабо искривленных сверхзвуковых профилей в компрессорах низкого давления и вентиляторах ТРДД. Авторами показано, что на пересечении траектории смещения номинальной точки «0» для решеток профилей и линейной зависимости для решетки плоских пластин определяется номинальная точка последней. На этом основании расширено семейство кривых, определяющих номинальные режимы «0» для профилей с нулевой и отрицательной кривизной $(0<\theta$, что позволяет строить характеристики турбинных решеток).

Полная характеристика решетки профилей, лопаточного венца, ступени и каскада включает изменение изоэнтропического напора и связанной с ним степени повышения давления. Авторами показано, что для построения характеристики решетки в виде коэффициента изо- 
энтропического напора $\bar{H}_{s}=f\left(\bar{c}_{a}\right)$ следует повернуть вокруг точки $\left\{\bar{H}_{T y}, 0\right\}$ лучи (участки до и после срыва) в базовой характеристике $\bar{H}_{T}=f\left(\bar{c}_{a}\right)$. Поворот определяется координатами точки «0» и значением $\eta_{0}$ КПД на номинальном режиме. При этом поправкой $\varphi_{m}$ учитывается искривление в зоне «m-0» (описывается функцией Безье). Учитывается также ступенчатый участок (при срыве) в базовой линии $\bar{H}_{T}=f\left(\bar{c}_{a}\right)$. Кроме того, добавляются поправки (выделенные на основе [2]), учитывающие скорость натекания на решетку $\lambda_{w 1}$ в виде

$$
\varphi_{T}\left(\frac{\bar{c}_{a}}{\bar{c}_{a 0}}, \lambda_{w 1}\right) \text { и } \varphi_{s}\left(\frac{\bar{c}_{a}}{\bar{c}_{a 0}}, \lambda_{w 1}\right) .
$$

На этом основании предложено параметризованное описание характеристики решетки профилей (которое в определенной степени пригодно и для лопаточного венца, ступени и каскада) для осевого случая (без учета диагональности и «просадки» осевой скорости) в виде

$$
\bar{H}_{T}=\bar{H}_{T y(1,2)}\left(1-\frac{\bar{c}_{1 a}}{\tilde{c}_{a x}}+\frac{\varphi_{T}}{0,75}+\varphi_{m}\right) .
$$

Здесь учтено, что поправки получены из кривых $K_{1}$ и $K_{2}$ Л.Е. Ольштейна, для которых $\bar{H}_{T y}=0,75$. Кроме того, авторами введено новое для лопаточных машин обозначение $\tilde{c}_{a x}=\frac{1}{\operatorname{ctg} \beta_{x}+\operatorname{ctg} \alpha_{1}}$. Оно позволяет записать также $\bar{H}_{T}=1-\frac{\bar{c}_{1 a}}{\tilde{c}_{a 2}}$, где $\tilde{c}_{a x}=\frac{1}{\operatorname{ctg} \beta_{2}+\operatorname{ctg} \alpha_{1}}$. Соответственно, для коэффициента изоэнтропического напора параметризованная зависимость имеет вид

$$
\bar{H}_{s}=\bar{H}_{T y(1,2)}\left(1-\frac{\bar{c}_{1 a}}{\tilde{c}_{a x s}}+\frac{\varphi_{s}}{0,75}+\varphi_{m}\right), \quad \text { где } \tilde{c}_{a x s}=\frac{\tilde{c}_{a x}}{1-\frac{\bar{H}_{T 0}}{\bar{H}_{T y 1}} \eta_{0}} .
$$

При этом для $\bar{c}_{a}>\bar{c}_{a(2,3)}$ (до срыва) используется значение $\bar{H}_{T y 1}$, а при $\bar{c}_{a}>\bar{c}_{a(2,3)}$ (в зоне срыва) используется значение $\bar{H}_{T y 2}$. 
Показано, что в координатах $H_{T}=f\left(\lambda_{1 a}, \lambda_{u}\right)$ каждая напорная ветвь $\lambda_{u}=$ const имеет качественно такой же вид, что и $\bar{H}_{T}\left(\bar{c}_{1 a}, \lambda_{u}\right)-$ характеристика коэффициента теоретического напора: $H_{T}=\bar{H}_{T}\left(\bar{c}_{1 a}, \lambda_{u}\right) \cdot \frac{2 k}{k+1} R T_{1}^{*} \lambda_{u}^{2}$. При этом в каждой точке напорной ветви $\lambda_{1 a}=\bar{c}_{1 a} \cdot \lambda_{u}$. Поэтому параметризация любой напорной ветви в этих координатах имеет вид $H_{T}=\frac{2 k}{k+1} R T_{1}^{*} \lambda_{u}^{2} \cdot \bar{H}_{T y(1,2)} \cdot\left(1-\frac{\lambda_{1 a}}{\lambda_{1 a x}}+\frac{\varphi_{T}}{0,75}+\varphi_{m}\right)$, где $\lambda_{1 a x}=\bar{c}_{1 a x} \cdot \lambda_{u}$.

Аналогично напорная ветвь изоэнтропического напора $H_{s}=\bar{H}_{s}\left(\bar{c}_{1 a}, \lambda_{u}\right) \cdot \frac{2 k}{k+1} R T_{1}^{*} \lambda_{u}^{2}$ параметризуется в виде

$$
H_{s}=\frac{2 k}{k+1} R T_{1}^{*} \lambda_{u}^{2} \cdot \bar{H}_{T y(1,2)} \cdot\left(1-\frac{\lambda_{1 a}}{\lambda_{\text {laxs }}}+\frac{\varphi_{s}}{0,75}+\varphi_{m}\right)
$$

Показано, что в отличие от работ [4-6] протекание границы помпажа для решетки, лопаточного венца и ступени определяется не точкой «m» (где достигается $\Delta \beta_{\max }$ и $\bar{H}_{T}=\bar{H}_{T m}-$ не обязательно максимальное значение), а точкой «ms» (где $\bar{H}_{s}=\bar{H}_{s m}$ ), при этом в общем случае $\bar{c}_{1 a m} \neq \bar{c}_{1 a m s}$. Граница помпажа «Г» при этом параметризуется в виде

$$
H_{s \Gamma}=\frac{\bar{H}_{s m}\left(\bar{c}_{1 a m s}, \lambda_{u}\right)}{\left(\bar{c}_{1 a m s}\right)^{2}} \cdot \frac{2 k}{k+1} R T_{1}^{*} \lambda_{1 a}^{2}
$$

Аналогично строятся и параметризуются на характеристике изолинии «0» (номинальных режимов) и «opt» (оптимальных режимов) как для изоэнтропического, так и теоретического напоров. Показано, что таким же образом могут быть построены характеристики лопаточного венца, ступени, каскада.

Например, после интегрирования характеристик решеток по высоте ЛВ (лопаточного венца) это позволяет построить характеристику РК (рабочего колеса) в традиционном виде, с учетом того, что $\pi_{\kappa}^{*}=\left(\frac{H_{s}}{c_{p} T_{1}^{*}}+1\right)^{\frac{k}{k-1}}$ с указанием границы помпажа «г», с нанесением 
изолиний «0», «opt». Аналогичным образом это реализуется для ступени, а при выделении характеристики эффективной ступени и для каскада (многоступенчатого компрессора).

Предложена методика, позволяющая строить на характеристике решетки, РК, ступени и многоступенчатого компрессора лини «ms», «0» и «opt» (границы помпажа, номинальных и оптимальных режимов). Например, для построения на характеристике ступени или компрессора линии номинальных режимов «0» достаточно для каждой напорной ветви повернуть базовую линию теоретического напора $\bar{H}_{T y}$ вокруг точки $\left\{H_{s}=f\left(\lambda_{1} a\right), 0\right\}$ на оси ординат до касания с линией $\bar{H}_{s}=f\left(\bar{c}_{a}\right)$.

Показано, что при проектировании газотурбинного двигателя и компрессора в его составе следует обеспечить протекание линии рабочих режимов в секторе между линией номинальных режимов «0» и границей помпажа (с учетом дополнительно нанесенной линии запирания «3»).

Для учета диагональности и «просадки» скорости выведена формула

$$
\bar{H}_{T}=\bar{r}_{2}^{2}\left(1-\frac{c_{2 a}}{c_{1 a}}\right)+\frac{c_{2 a}}{c_{1 a}} \bar{H}_{T \mathrm{oc}}
$$

Здесь, в отличие от известных методов, предложено обозначать компоненту вдоль поверхности тока в меридиональной плоскости $c_{a}$; $\bar{H}_{T \text { ос }}$ - коэффициент напора эквивалентной осевой решетки (без «просадки») с эффективным среднемиделевым шагом. При этом параметр $\bar{r}_{2}=\frac{r_{2}}{r_{1}}$ характеризует диагональность (в том числе центробежное и радиальное течение), $\frac{c_{2 a}}{c_{1 a}}$ характеризует «просадку» скорости вдоль поверхности тока в меридиональной плоскости.

Кроме того, авторами предложен метод параметризации характеристик решеток профилей (а также ступеней, компрессоров) при изменении закрутки на входе (положения - входной направляющий аппарат (ВНА) и направляющий аппарат (НА)). Показано, что при изменении закрутки на входе $\alpha_{1}$ номинальная точка «0» смещается по линии Хауэлла, при 
этом базовая линия на характеристике решетки не меняет свою форму, а лишь поворачивается вокруг точки $\left\{H_{T y}, 0\right\}$ на оси ординат. Наиболее наглядно такие преобразования показаны в координатах $H_{T}=f\left(\lambda_{1 a}, \lambda_{u}\right)$. При этом координата любой точки на характеристике вдоль оси абсцисс смещается в соответствии с $\lambda_{1 a}^{\prime}=\frac{\lambda_{u}}{\frac{\lambda_{u}}{\lambda_{1 a}}+\operatorname{ctg} \alpha_{1}}$, где $\lambda_{1 a}$ определено при $\alpha_{1}=90^{\circ}$. Исходя из этого в приведенных выше параметризованных описаниях напорных ветвей $\lambda_{\text {lax }}^{\prime}=\frac{\lambda_{u}}{\frac{\lambda_{u}}{\lambda_{1 a x}}+\operatorname{ctg} \alpha_{1}}$ и $\lambda_{\text {laxs }}^{\prime}=\frac{\lambda_{u}}{\frac{\lambda_{u}}{\lambda_{\text {laxs }}}+\operatorname{ctg} \alpha_{1}}$. Здесь также $\lambda_{1 a x}$ и $\lambda_{\text {laxs }}$ изначально определены при $\alpha_{1}=90^{\circ}$.

Разработанные алгоритмы положены в основу созданной авторами системы имитационного моделирования VENEC $[10,11]$. С ее помощью проведено моделирование ряда ступеней и компрессоров (в том числе с поворотным ВНА и центробежных).

\section{Заключение}

Предложенный метод позволяет получать характеристики в параметризованном виде лопаточных венцов и ступеней, включая зону срыва и запомпажные ветви. Он предоставляет возможность построить универсальную обезразмеренную характеристику компрессоров, включая универсальную границу помпажа, экстраполировать напорные ветви, строить новые, уточнять границу помпажа при экспериментах. Параметризованные характеристики решеток профилей, лопаточных венцов, ступеней и многоступенчатых компрессоров используются при выборе параметров, оптимизации при проектировании компрессоров, при моделировании изменения характеристик компрессоров в эксплуатации. Разработанные методы позволяют уточнить алгоритмы, используемые в существующих системах моделирования компрессоров. Они использованы в созданной авторами системе имитационного повенцового моделирования компрессоров различных типов VENEC. 


\section{Библиографический список}

1. Расчетная программа предварительного проектирования осевых компрессоров и турбин AXIAL [Электронный ресурс]. - URL: http://conceptsnrec.ru/software/axial (дата обращения: 01.11.2015).

2. Система имитационного моделирования COMPRESSOR: св-во № 2009612688 / Д.А. Ахмедзянов, А.Б. Козловская, И.А. Кривошеев. М.: Роспатент, 2009.

3. Емин О.Н., Карасев В.Н., Ржавин Ю.А. Выбор параметров и газодинамический расчет осевых компрессоров и турбин авиационных ГТД: учеб. пособие. - М.: Дипак, 2003. - 156 с.

4. Проектный термогазодинамический расчет основных параметров авиационных лопаточных машин / А.Н. Белоусов, Н.Ф. Мусаткин, В.М. Радько, В.С. Кузьмичёв. - Самара: Изд-во Самар. гос. аэрокосм. ун-та, 2006. - 316 с.

5. Хауэлл А.Р. Газодинамика осевого компрессора // Развитие газовых турбин: сб. стат.: пер. с англ. / под ред. В.Л. Александрова. - М.: Изд-во БНТИМАП, 1947. - С. 42-56.

6. Ольштейн Л.Е., Процеров В.Г. Метод расчета осевого компрессора по данным продувок плоских компрессорных решеток // Тр. ЦИАМ. 1948. - № 150. - С. 1-60.

7. Обобщение результатов продувок плоских компрессорных решеток методом регрессионного анализа / П.П. Казанчан, Б.В. Караваев, В.И. Серков, В.Н. Шишкин // Тр. ЦИАМ. - 1975. - № 679. - С. 1-64.

8. Бунимович А.И., Святогоров А.А. Аэродинамические характеристики плоских компрессорных решеток при большой дозвуковой скорости // Лопаточные машины и струйные аппараты: сб. ст. - М.: Машиностроение, 1967. - Вып. 2. - С. 5-35.

9. Бунимович А.И., Святогоров А.А. Обобщение результатов исследования плоских компрессорных решеток при дозвуковой скорости // Лопаточные машины и струйные аппараты: сб. ст. - М.: Машиностроение, 1967. - Вып. 2. - С. 36-66.

10. Система имитационного моделирования VENEC: св-во № 2012612817 / К.Е. Рожков, И.А. Кривошеев. - М.: Роспатент, 2012.

11. Кривошеев И.А., Рожков К.Е. Развитие методов анализа и расчета характеристик решеток профилей осевых компрессоров // Вестник ИрГТУ. - 2012. - № 2(61). - С. 26-32. 


\section{References}

1. Raschetnaya programma predvaritelnogo proektirovaniya osevykh kompressorov i turbin AXIAL [Computational program for preliminary design of axial compressors and turbines], available at: http://conceptsnrec.ru/software/axial (accessed 1 November 2015).

2. Akhmedzyanov D.A., Kozlovskaya A.B., Krivosheev I.A. Sistema imitatsionnogo modelirovaniya COMPRESSOR [The system of COMPRESSOR]. Certificate № 2009612688. Moscow: Rospatent, 2009.

3. Emin O.N., Karasev V.N., Rzhavin Yu.A. Vybor parametrov i gazodinamicheskiy raschet osevykh kompressorov i turbin aviatsionnykh GTD [Choice of parameters and gas-dynamic computation of axial compressors and aircraft turbines]. Moscow: Dipak, 2003. 156 p.

4. Belousov A.N., Musatkin N.F., Radko V.M., Kuzmichev V.S. Proektnyy termogazodinamicheskiy raschet osnovnykh parametrov aviatsionnykh lopatochnykh mashin [Designed termo-gaz-dynamic computation of general quantities of aircraft impeller machines]. Samarskiy gosudarstvennyi aerokosmicheskii universitet, 2006. 316 p.

5. Khouell A.R. Gazodinamika osevogo kompressora [Gas-dynamics of axial compressor]. Razvitie gazovykh turbin. Moscow, 1947, pp. 42-56.

6. Olshteyn L.E., Protserov V.G. Metod rascheta osevogo kompressora po dannym produvok ploskikh kompressornykh reshetok [The method of calculation of the axial compressor based on data of scavenging plane compressor grids]. Trudy Tsentralnogo instituta aviatsionnogo motorostroeniya, 1948, no. 150 , pp. 1-60.

7. Kazanchan P.P., Karavaev B.V., Serkov V.I., Shishkin V.N. Obobshchenie rezultatov produvok ploskikh kompressornykh reshetok metodom regressionnogo analiza [Generalization of the results of scavenging plane compressor grids by regression procedure]. Trudy Tsentralnogo instituta aviatsionnogo motorostroeniya, 1975, no. 679, pp. 1-64.

8. Bunimovich A.I., Svyatogorov A.A. Aerodinamicheskie kharakteristiki ploskikh kompressornykh reshetok pri bolshoy dozvukovoy skorosti [Aerodynamic characteristics of the plane compressor grids at high subsonic velocity]. Sbornik statey "Lopatochnye mashiny i struynye apparaty”. Moscow: Mashinostroenie, 1967, iss. 2, pp. 5-35.

9. Bunimovich A.I., Svyatogorov A.A. Obobshchenie rezultatov issledovaniya ploskikh kompressornykh reshetok pri dozvukovoy skorosti [Generalization of the research results of plane compressor grids at subsonic 
velocity]. Sbornik statey "Lopatochnye mashiny i struynye apparaty”. Moscow: Mashinostroenie, 1967, iss. 2, pp. 36-66.

10. Rozhkov K.E., Krivosheev I.A. Sistema imitatsionnogo modelirovaniya VENEC [System of the simulation modeling VENEC]. Certificate № 2012612817. Moscow: Rospatent, 2012.

11. Krivosheev I.A., Rozhkov K.E. Razvitie metodov analiza i rascheta kharakteristik reshetok profiley osevykh kompressorov [Development of the analysis and computational techniques for characteristics of profile grids of axial compressors]. Vestnik Irkutskogo gosudarstvennogo tekhnicheskogo universiteta, 2012, no. 2(61), pp. 26-32.

\section{Об авторах}

Кривошеев Игорь Александрович (Уфа, Россия) - доктор технических наук, профессор кафедры «Авиационные двигатели» Уфимского государственного авиационного технического университета (45000, г. Уфа, ул. К. Маркса, д. 12, e-mail: krivosh@sci.ugatu.ac.ru).

Рожков Кирилл Евгеньевич (Уфа, Россия) - ассистент кафедры «Авиационная теплотехника и теплоэнергетика» Уфимского государственного авиационного технического университета $(45000$, г. Уфа, ул. К. Маркса, д. 12, e-mail: rke85@mail.ru).

Симонов Николай Борисович (Уфа, Россия) - аспирант кафедры «Авиационные двигатели» Уфимского государственного авиационного технического университета (45000, г. Уфа, ул. К. Маркса, д. 12, e-mail: sventigo@yandex.ru).

\section{About the authors}

Igor A. Krivosheev (Ufa, Russian Federation) - Doctor of Technical Sciences, Professor, Department of Aircraft Engines, Ufa State Aviation Technical University (12, K. Marx st., Ufa, 45000, Russian Federation, e-mail: krivosh@sci.ugatu.ac.ru).

Kirill E. Rozhkov (Ufa, Russian Federation) - Assistant, Department of Thermal Engineering and Aviation Power System, Ufa State Aviation Technical University (12, K. Marx st., Ufa, 45000, Russian Federation, e-mail: rke85@mail.ru).

Nikolay B. Simonov (Ufa, Russian Federation) - Postgraduate Student, Department of Aircraft Engines, Ufa State Aviation Technical University (12, K. Marx st., Ufa, 45000, Russian Federation, e-mail: sventigo@yandex.ru).

Получено 26.09.2015 